RESEARCH ARTICLE

\title{
High-power non-perturbative laser delivery diagnostics at the final focus of 100-TW-class laser pulses
}

\author{
Fumika Isono $^{1,2}$, Jeroen van Tilborg ${ }^{1}$, Samuel K. Barber ${ }^{1}$, Joseph Natal ${ }^{1,2}$, Curtis Berger ${ }^{1,2}$, Hai-En Tsai ${ }^{1}$, \\ Tobias Ostermayr ${ }^{1}$, Anthony Gonsalves ${ }^{1}$, Cameron Geddes ${ }^{1}$, and Eric Esarey ${ }^{1}$ \\ ${ }^{1}$ Lawrence Berkeley National Laboratory, Berkeley, CA, USA \\ ${ }^{2}$ University of California, Berkeley, CA, USA \\ (Received 16 December 2020; revised 8 February 2021; accepted 26 February 2021)
}

\begin{abstract}
Controlling the delivery of multi-terawatt and petawatt laser pulses to final focus, both in position and angle, is critical to many laser applications such as optical guiding, laser-plasma acceleration, and laser-produced secondary radiation. We present an online, non-destructive laser diagnostic, capable of measuring the transverse position and pointing angle at focus. The diagnostic is based on a unique double-surface-coated wedged-mirror design for the final steering optic in the laser line, producing a witness beam highly correlated with the main beam. By propagating low-power kilohertz pulses to focus, we observed spectra of focus position and pointing angle fluctuations dominated by frequencies below $70 \mathrm{~Hz}$. The setup was also used to characterize the excellent position and pointing angle correlation of the $1 \mathrm{~Hz}$ high-power laser pulses to this low-power kilohertz pulse train, opening a promising path to fast non-perturbative feedback concepts even on few-hertz-class high-power laser systems.
\end{abstract}

Keywords: high-power lasers; laser diagnostics; laser stabilization

\section{Introduction}

High-power laser systems, targeting science at relativistic laser intensities $\left(>10^{18} \mathrm{~W} / \mathrm{cm}^{2}\right)$, are now routinely employed at laboratories all over the world, ranging from peak powers of tens of terawatts to multi-petawatt and beyond ${ }^{[1-4]}$. Such high peak power is enabled by concentrating joules of laser energy into femtosecond-duration pulses via chirped pulse amplification ${ }^{[5]}$. When focused to micrometer-sized spots, the peak intensity reaches a regime where matter is easily ionized and the electrons quiver at relativistic speeds. Nonlinear laser-plasma processes can then be accessed, driving applications such as laser-plasma acceleration (LPA; where plasma electrons are accelerated to gigaelectronvolt-level energies over distances of just centimeters ${ }^{[6]}$ ), LPA-based light sources ${ }^{[7-10]}$, plasma-based X-ray lasers ${ }^{[11]}$, and ion acceleration from solid targets ${ }^{[12]}$, among others.

The nonlinear physics at play in the high-power laserplasma interaction makes applications highly sensitive to

Correspondence to: J. van Tilborg, Lawrence Berkeley National Laboratory, Berkeley, CA 94720, USA. Email: JvanTilborg@lbl.gov laser alignment. For example, transverse position jitter at focus of the order of $10 \mu \mathrm{m}$ is problematic when shortscale plasma density features are present, when colliding or overlapping with multiple micrometer-sized laser foci ${ }^{[13-16]}$, or when guiding intense laser pulses in plasmas ${ }^{[17,18]}$. Similarly, the pointing angle of the laser axis affects the coupling of the laser-plasma-produced secondary radiation (electron beams, X-rays, etc.) onto downstream beam and radiation optics such as quadrupole magnets. Hence, control of the position, pointing angle, and other laser parameters are key to high-quality laser applications.

Over recent years, LPA-relevant pioneering work has been performed on pointing correction ${ }^{[19]}$ and wavefrontoptimizing feedback loops ${ }^{[20]}$. Traditionally, high-power transverse focal position and angle are measured by monitoring the leakage of the last optic in the beam line ${ }^{[21]}$. However, because the leakage beam is transmitted through the mirror, vibrations and thermal motion of that specific mirror are not picked up by the leakage beam. In particular, with long-focal-length geometries, the vibrations on this last optic can translate into large position fluctuations at focus, uncorrelated to the leakage beam. In this paper, past 
limitations are overcome by the installation of a special final steering mirror (the last optic delivering the converging laser beam from the parabola towards focus), where its backsurface reflection of the main beam is used to provide online non-perturbative monitoring of the high-power laser focus position and angle in a two-camera setup. We refer to the beam reflected from the back surface as the 'witness beam.' This witness beam is a fully correlated copy of the high-power beam because it shares exactly the same mirror reflections.

In addition, such a witness beam diagnostic also lends itself to integration of pointing stabilization based on feedback concepts. As part of this paper we present data taken to quantify the system's focal position and pointing angle fluctuations, by using both the low-power ( $1 \mathrm{~mJ}$ at focus) high-repetition-rate $(1 \mathrm{kHz})$ laser pulse train, as well as the low-repetition-rate $(1 \mathrm{~Hz})$ high-power $(\sim 2.5 \mathrm{~J}$ at focus $)$ laser pulses. The low-power kilohertz pulse train is derived from the laser front-end. As both pulse trains originate from the same pulse train at upstream, with moderate thermal lensing of the amplifier crystals and kilohertz pulse having enough energy to be propagated, they have roughly the same beam size and the divergence through the whole system. This makes the low-power kilohertz pulse train well suited as the alignment beam. It carries the same vibrations as the high-power beam, from the source through the entire laser system, all the way to focus, which is not possible if the alignment beam is injected after amplifiers ${ }^{[19]}$. By propagating the low-power kilohertz pulses to focus, we observed focus position and pointing angle fluctuation dominated by frequencies below $70 \mathrm{~Hz}$ (consistent with spectra of equipment vibrations, air turbulence, among other effects). This encouraging finding supports the vision that if highpower laser systems were to become available at kilohertz repetition rates (which is currently being pursued by various groups $^{[22,23]}$ ), fast feedback mechanisms using the witness beam technique demonstrated in this paper could be able to correct for the less than $70 \mathrm{~Hz}$ motion.

While awaiting such high-power kilohertz laser systems, we study the option of using the non-amplified kilohertz background pulse train to correct beam misalignments for the $1 \mathrm{~Hz}$ amplified high-power pulses. By simultaneously using two laser diagnostics (the witness beam diagnostic as well as a downstream mode-imager diagnostic), we measured the position and pointing angle correlation between the $1 \mathrm{~Hz}$ amplified pulses and the $1 \mathrm{kHz}$ non-amplified pulse train. One key question we aimed to answer is whether the $1 \mathrm{~Hz}$ amplified pulses have a decreased correlation to temporally adjacent kilohertz background pulses owing to the $1 \mathrm{~Hz}$ amplification process (i.e., whether the pump laser and amplification process impart a temporary uncorrelated positional and angular kick to the $1 \mathrm{~Hz}$ amplified laser pulse). If that were the case, it would critically limit the option for fast feedback at few-hertz laser systems by using the non-amplified background laser. However, it will be presented that full-power measurements confirmed that the amplification process did not add a measurable uncorrelated contribution to the $1 \mathrm{~Hz}$ amplified pulses.

The work presented here demonstrates a very accurate non-perturbative high-power laser diagnostic, allowing shottagged laser delivery information on position and angle. The demonstrated correlation of the high-power laser pulse train to the non-amplified background pulse train highlights the option for feedback integration at bandwidths larger than the $<70 \mathrm{~Hz}$ environmental fluctuations. This would allow significant improvements in stability, quality, and applicability of high-power laser systems.

\section{Experimental setup}

A schematic of the 100-TW-class laser system and online target chamber monitoring setup is shown in Figure 1. The titanium-sapphire-based chirped-pulsed-amplification laser system was built to serve the LPA-based Free Electron Laser project at the BELLA Center ${ }^{[24]}$. It has the capability to approach $100 \mathrm{TW}$-class peak power based on $\simeq 2.5 \mathrm{~J}$ energy on target (3.8 J before the compressor) and 35 fs FWHM (full width at half maximum) pulse duration.

The commercial $1 \mathrm{kHz}, 800 \mathrm{~nm}$ front-end laser is based on the Vitara oscillator from Coherent ${ }^{[25]}$ coupled with the Legend Elite regenerative amplifier. The laser pulses contain $3 \mathrm{~mJ}$ and are stretched from 35 fs to $290 \mathrm{ps}$ at the stretcher to prevent damage to optics during amplification. The pulse train is then propagated to amplifier 2 (a four-pass bowtie amplifier), amplifier 3 (a three-pass bow-tie amplifier), and to amplifier 4 (a three-pass bow-tie amplifier). All three amplifier crystals are pumped by the same $16 \mathrm{~J} /$ shot $5 \mathrm{~Hz}$ pump laser (GAIA from Thales ${ }^{[26]}, 532 \mathrm{~nm}, \sim 16 \mathrm{~ns}$ pulse duration). The amplified pulse energy reaches $100 \mathrm{~mJ}$ after amplifier 2, $1 \mathrm{~J}$ after amplifier 3, and $4 \mathrm{~J}$ after amplifier 4, whereas its size increases from $6 \mathrm{~mm}$ FWHM to $12 \mathrm{~mm}$ before amplifier 3 , and to $23 \mathrm{~mm}$ before amplifier 4 . All Ti:sapphire crystals are water cooled.

Although the repetition rate (as set by the pump lasers) can operate up to $5 \mathrm{~Hz}$, in this study we operated the pump laser at $1 \mathrm{~Hz}$ repetition rate. In this mode, the pulse train coming out of the amplifiers consists of amplified pulses separated in time by $1 \mathrm{~s}$, and a train of unamplified background pulses separated by $1 \mathrm{~ms}$. With the pump laser blocked, the remnant $1 \mathrm{kHz}$ pulse train leaving the front-end system will be labeled as the ' $1 \mathrm{kHz}$ non-amplified beam.' Following the three amplifiers, the laser pulses are spatially expanded from 23 to $81 \mathrm{~mm}$ FWHM using the reflective-curved-mirror telescope with a magnification of $3.5 \times$. After being routed into the vacuum system, the pulses are compressed to $35 \mathrm{fs}$ (FWHM) with a grating compressor, and focused into the target chamber using an off-axis parabolic mirror (OAP) with focal length of $3 \mathrm{~m}$. As shown in Figure 1, at $1 \mathrm{~m}$ 


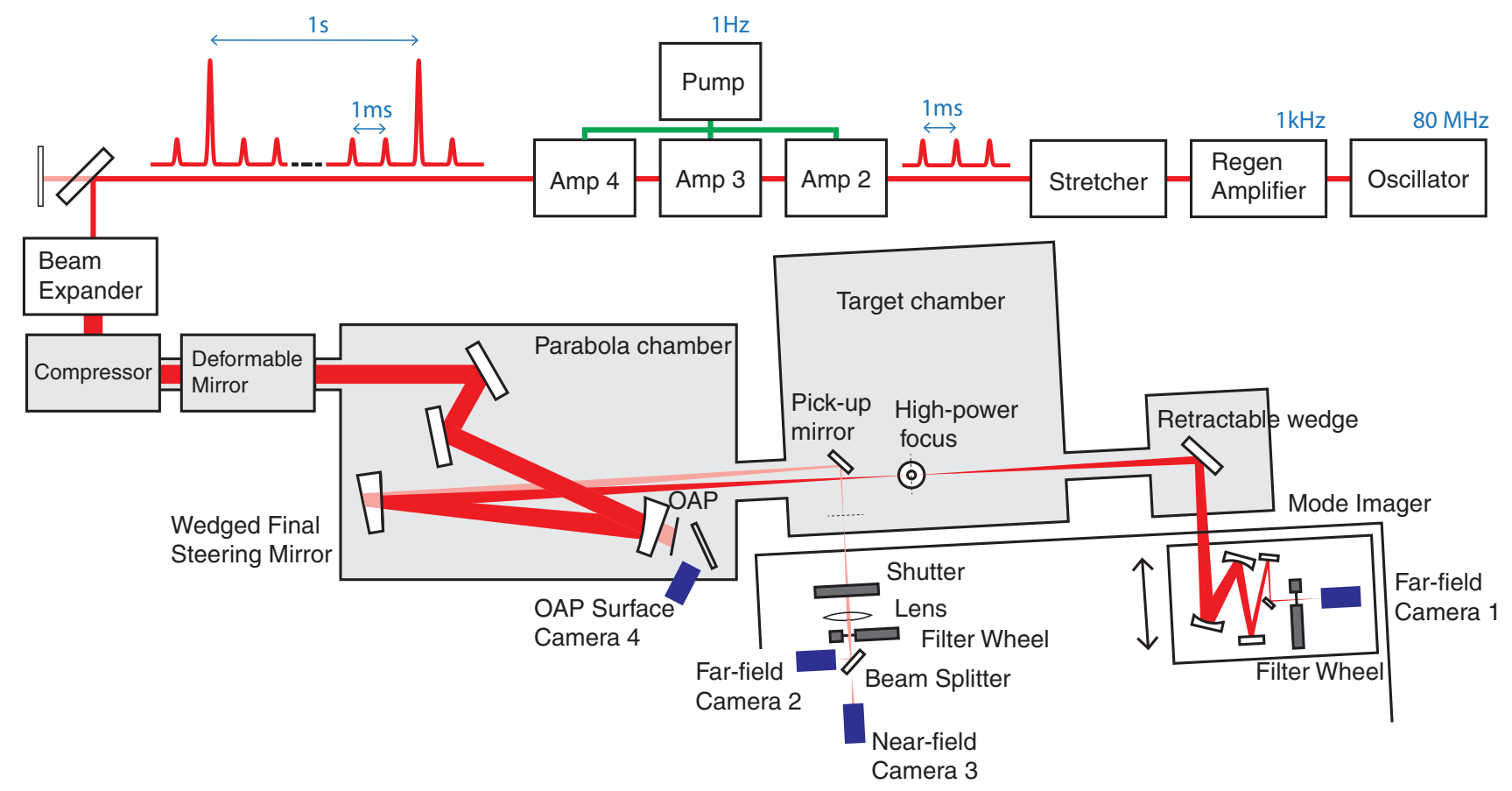

Figure 1. Experimental setup of the 100-TW-class laser system. A $1 \mathrm{kHz}, 800 \mathrm{~nm}, 35 \mathrm{fs}$ laser pulse train is produced in the regenerative amplifier (Regen) and stretched to $300 \mathrm{ps}$, and then every one in a thousand pulses is amplified in three successive multi-pass bow-tie amplifiers (Amp 2, 3, and 4). A single commercial pump laser $(532 \mathrm{~nm}, \sim 16 \mathrm{~ns}$ pulse duration) is routed to all three amplifier Ti:sapphire crystals with $1 \mathrm{~Hz}$ repetition rate. The laser pulses are then compressed to $35 \mathrm{fs}$ and focused into the target chamber, where a gas jet is placed for LPA experiments. The laser mode at focus is measured with two diagnostics: (1) a common mode imager (after insertion of the retractable wedge) with camera 1 recording the far-field laser profile by imaging the target chamber focus plane; and (2) a correlated witness beam setup, where a back-surface-reflected final steering mirror is routing a correlated copy of the main beam to a setup measuring the laser's far-field (camera 2, imaging the target chamber focus plane) and quasi-near-field (camera 3 , imaging a plane $44 \mathrm{~mm}$ downstream of the target chamber focus). The near-field beam profile at the off-axis parabolic mirror (OAP) surface is recorded by camera 4 . Gray boxes represent vacuum chambers.

downstream of the OAP a final steering mirror directs the converging beam to final focus.

The laser mode on target is measured by inserting an uncoated retractable wedge downstream of focus (hence 'destructive' to the path of electron beam propagating downstream), and routing the beam through a mode imager setup consisting of curved reflective mirrors and two more uncoated wedge reflections for appropriate attenuation onto far-field CCD camera 1 (Basler acA1300-22gm, 1.3 MP resolution, pixel size $3.75 \mu \mathrm{m} \times 3.75 \mu \mathrm{m}$, pixel bit depth 12 bits). Camera 1 is thus imaging the plane of the focused beam in the target chamber at magnification $1.5 \times$.

In addition to the mode imager setup, an alternative laser delivery monitor was developed. The copy of the high-power main beam was generated by the main beam reflected at the rear surface of the final steering mirror, which is wedged at an angle of $0.25^{\circ}$, has a $98 \%$ surface reflectivity on the front surface, and $80 \%$ reflectivity on the rear surface. Thus, $2 \% \times 80 \% \times 2 \%=0.032 \%$ of the main beam was reflected from the rear surface of the wedge. This copy of the main beam will be labeled as the 'witness beam.' The transmission of this mirror, was used for laser alignment and the measurement of its energy. At the front surface of the wedge, the reflected witness beam was separated from the main beam by $1.0 \mathrm{~mm}$ and $0.76^{\circ}$. These parameters depend on the angle of incidence onto the wedge $\left(4.4^{\circ}\right)$, the thickness $(9.5 \mathrm{~mm})$, and the material (fused silica). The witness beam $B$-integral, which is a measure of the nonlinear phase shift of light as it propagates through the final steering mirror, was calculated to be $0.41 \mathrm{rad}$, which is low enough to keep unwanted effects such as self-phase modulation and self-focusing at acceptable levels (for a thicker optic and/or increased laser energy this effect should be re-evaluated). The aberrations introduced to the converging witness beam by the doublepass through the wedged mirror are expected to be minor due to the small wedge angle, its sub-centimeter thickness, and the near-normal angle of incidence of the incoming laser. This assumption is supported by the fact that the main beam and the witness beam have similar beam sizes at focus, with no sign of significant aberrations, as shown in Figures 2(a) and 2(b) and accompanying text in Section 3. The transverse position separation between the main beam and witness beam at the target chamber focal plane was $27 \mathrm{~mm}$. The witness beam was reflected by a rigidly mounted reflective mirror positioned $8 \mathrm{~cm}$ upstream of the witness beam focus. Thus, the additional fluctuations of the witness beam picked up by this mirror are small compared with the last optic shared by the main beam and the witness beam. A vacuum 


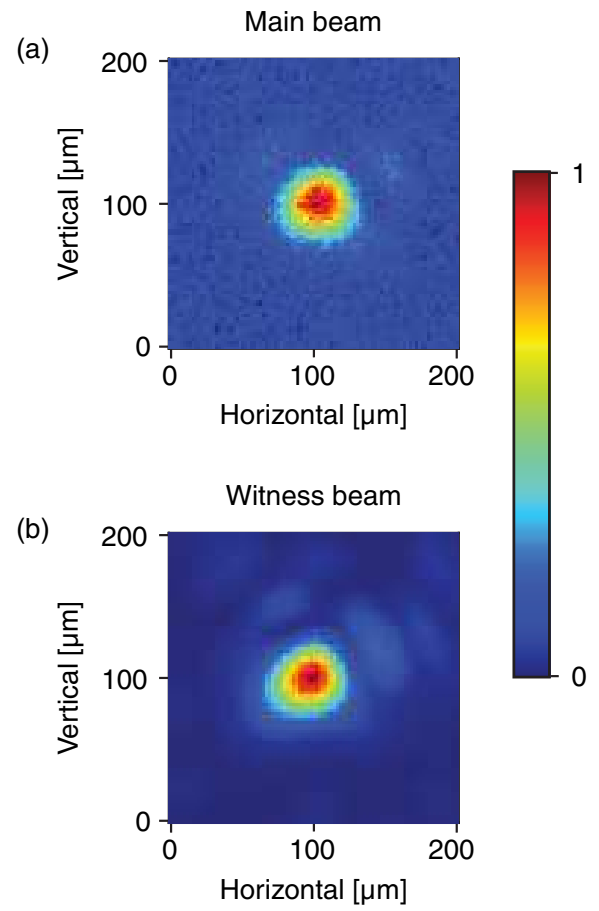

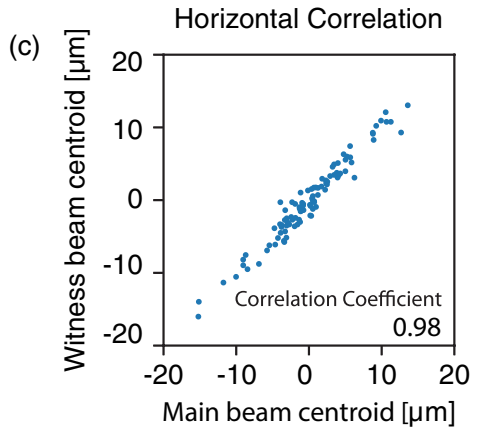

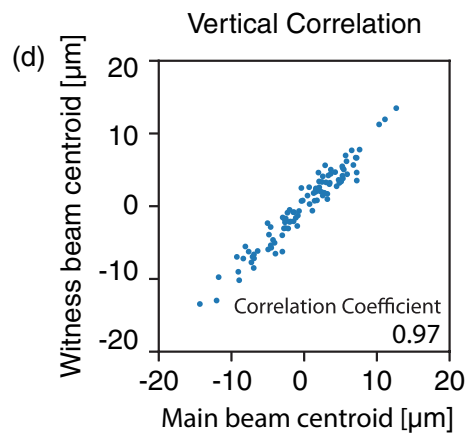

Figure 2. A single-shot transverse mode profile of the amplified beam captured by (a) camera 1 (mode imager) and (b) camera 2 (witness beam). (c), (d) Correlation of 100 consecutive high-power shots (at $1 \mathrm{~Hz}$ ) of the main beam and witness beam centroids in horizontal and vertical direction, respectively.

window $11 \mathrm{~cm}$ after the witness beam focus transmitted the beam onto an achromatic lens and a beamsplitter. The reflected beam was captured by far-field camera 2 (imaging the witness beam focus plane), whereas the transmitted beam was sent onto quasi-near-field camera 3 (imaging a plane $44 \mathrm{~mm}$ downstream of the witness beam focal plane). CMOS cameras 2 and 3 (Basler acA720-290gm, 0.4 MP resolution, pixel size $6.9 \mu \mathrm{m} \times 6.9 \mu \mathrm{m}$ ) were operated in $1 \mathrm{kHz}$ frame rate by reducing the region of interest $(\mathrm{ROI})$ to $100 \times 100$ pixels. The magnification of this imaging system was $1.1 \times$. Pixel bit depth of all four cameras presented here was set to 12 bits. When recording images, the signal level was typically set to a factor of $\times 1000$ above the noise, which was approximately three counts. We typically threshold the image right above the background which varied between 20 and 200 counts depending on cameras and the laser power. It was verified that changing the threshold conditions above the background did not have a noticeable impact on the correlation studies presented in this paper. As the witness beam setup can monitor both the position of the transverse focus (centroid on camera 2) and the angle of the focal line (centroid difference between cameras 2 and 3 divided by separation), this setup was capable of measuring the laser delivery non-destructively without interfering with the high-power laser focus and the LPA electron beam transport line. Filter wheels containing thin pellicle attenuators were installed in front of the mode imager and witness beam cameras. Both systems were mounted on an optical table isolated from the vacuum chambers for better stability.

\section{Witness beam validation in high-power mode}

To measure the correlation between the two diagnostics and to compare the main beam and the witness beam, synchronized far-field (focus) images on camera 1 (mode imager) and camera 2 (witness beam) were captured. Figure 2(a) shows a far-field mode of the main beam captured with camera 1, whereas Figure 2(b) shows a far-field mode of a witness beam captured with camera 2 . The beam size measured with camera 1 was $46 \mu \mathrm{m}$ FWHM. The mode imager point spread function (PSF), arising from a limited collected angle, was measured by temporarily placing a single-mode fiber tip at the target chamber focal plane (mode size less than $5 \mu \mathrm{m}$, see Figure 1). This measured PSF indicates a resolution limit of $25 \mu \mathrm{m}$ FWHM, thus yielding a PSF-deconvolved target chamber focus beam size of $\sqrt{46^{2}-25^{2}}=39 \mu \mathrm{m}$ FWHM. In the witness beam diagnostic (not constrained by a limited collection angle), the size of the witness beam measured with camera 2 was $40 \mu \mathrm{m}$ FWHM, providing agreement with the mode imager setup.

Figures 2(c) and 2(d) show the centroid correlations of 100 consecutive shots (with each shot $n$ yielding a centroid $c_{n}$ ) of the amplified main and witness beams, in the horizontal and vertical planes, respectively. Note that each camera was calibrated to incorporate the transverse magnification at the imaged plane (the focal plane inside the target chamber). The standard deviation of 100 collected centroid measurements $\sigma_{c}$ on the centroids on the mode imager is $5.4 \mu \mathrm{m}$ (root mean square) in horizontal direction and $5.3 \mu \mathrm{m}$ in vertical 
direction, corresponding approximately to an angle-ofincidence fluctuation of the beam onto the fixed point on the OAP of $1.8 \mu \mathrm{rad}$ in horizontal direction and $1.9 \mu \mathrm{rad}$ in vertical direction (not to be confused with the pointing angle fluctuations of the post-OAP target-line laser axis where the target position is fixed).

The witness-to-main beam Pearson's correlation coefficient (measuring the degree of correlation) for these 100 shots (100 s of $1 \mathrm{~Hz}$ acquisition), as shown in Figures 2(c) and 2(d), was 0.98 and 0.97 in horizontal and vertical direction, respectively. Here, the Pearson's correlation coefficient is defined as

$$
\rho=\frac{\operatorname{cov}\left(c_{n 1}, c_{n 2}\right)}{\sigma_{c 1} \sigma_{c 2}},
$$

with $\operatorname{cov}\left(c_{n 1}, c_{n 2}\right)$ being the covariance of the two centroid measurements $c_{n 1}$ (mode imager camera 1) and $c_{n 2}$ (witness camera 2), and $\sigma_{c 1}$ and $\sigma_{c 2}$ their respective standard deviations. The standard deviation of the residual centroid fluctuations (uncorrelated) $\sigma_{\text {res }}$ was found to be $1.2 \mu \mathrm{m}$ in horizontal direction and $1.3 \mu \mathrm{m}$ in vertical direction. Note that both main and witness beams share exactly the same mirror reflections all the way to the final steering mirror, limiting sources of uncorrelated factors. Camera 2 was imaging a plane $8 \mathrm{~cm}$ downstream of the reflecting mirror inside the target chamber, thus making it relatively insensitive to vibrations of this optic. Most residual fluctuation can be attributed to the fact that the mode imager has a series of post-focus reflective optics. We studied this post-focus by placing a rigidly mounted single-mode fiber tip at the target chamber focus location, which was also used to measure the PSF. We found the centroid fluctuation $\sigma_{c}$ to be $0.84 \mu \mathrm{m}$ in horizontal direction and $1.2 \mu \mathrm{m}$ in vertical direction, thus accounting for most of the witness-to-main beam residual fluctuation $\sigma_{\text {res }}$.

(a)
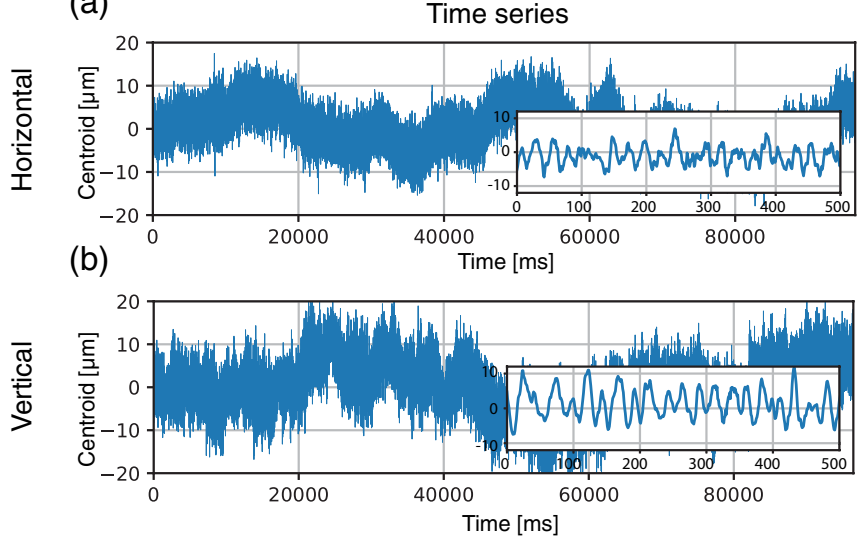

\section{Non-amplified kilohertz beam focus position and pointing angle fluctuations}

To investigate the source of pointing fluctuations in the $1 \mathrm{~Hz}$ amplified beam, we blocked the pump laser to the main amplifiers, and studied the non-amplified kilohertz pulse train focus position and pointing angle fluctuations. The farfield mode of the witness beam was recorded on camera 2 for $100 \mathrm{~s}$ at $1 \mathrm{kHz}$ frame rate $(N=100,000$ shots in total), with the centroids plotted in Figures 3(a) and 3(b). The standard deviation was $5.0 \mu \mathrm{m}$ in horizontal direction and $6.5 \mu \mathrm{m}$ in vertical direction, which is similar to the high-power fluctuations in Figure 2. This indicates that amplifying the beam does not increase fluctuation. Hence, within the resolution of our measurements, observed pointing fluctuations in the amplified $1 \mathrm{~Hz}$ beam can likely be corrected by steering mirrors based on fluctuations of non-amplified pulse train. Figures 3(c) and 3(d) show the intensity $|2 I(f) / N|$ of the discrete Fourier transform $I(f)$ of centroids $c_{n}$, calculated together with the power spectral density $S(f)$ following

$$
S(f)=\frac{\Delta t^{2}}{T}|I(f)|^{2}=\frac{\Delta t^{2}}{T}\left|\sum_{n=1}^{N} c_{n} e^{-2 i \pi f n \Delta t}\right|^{2},
$$

with $N$ total number of shots, $\Delta t=1 \mathrm{~ms}$ the time between centroid acquisitions, and $T=N \Delta t$ the total acquisition period. The bulk of the frequency components of the fluctuations are below $100 \mathrm{~Hz}$, which we predict could be eliminated by an active control stabilization system using the $1 \mathrm{kHz}$ nonamplified beam.

Similarly to Figure 3, we recorded the optical axis angle $\theta_{n}$ near final focus, by calculating the difference between the beam centroids as measured by cameras 2 and 3 , and by dividing it by the longitudinal separation in imaged planes. Figures 4(a) and 4(b) show the time series of the optical

(c)

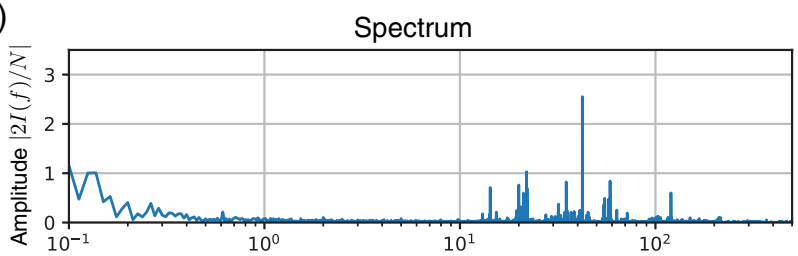

(d)

Frquency $f[\mathrm{~Hz}]$

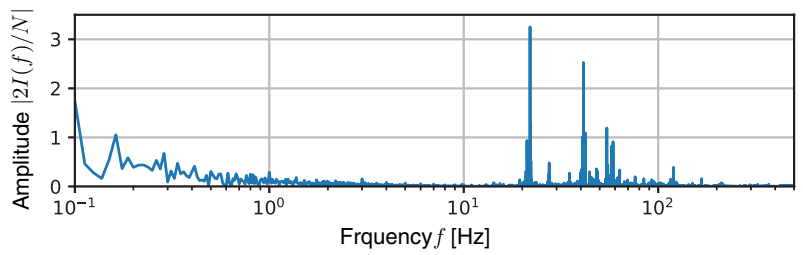

Figure 3. (a), (b) Time series of $1 \mathrm{kHz}$ non-amplified beam centroids in horizontal and vertical directions measured with far-field witness beam camera 2 . The inset displays a zoomed-in sub-set of the first 500 shots. (c), (d) The amplitude $|2 I(f) / N|$ of the discrete Fourier transform $I(f)$ of the time series (a) and (b), respectively, with $N=100,000$ the number of samples. In addition to sub-hertz drifts, several peaks in the fluctuation spectrum can be observed between 10 and $100 \mathrm{~Hz}$. 
(a)
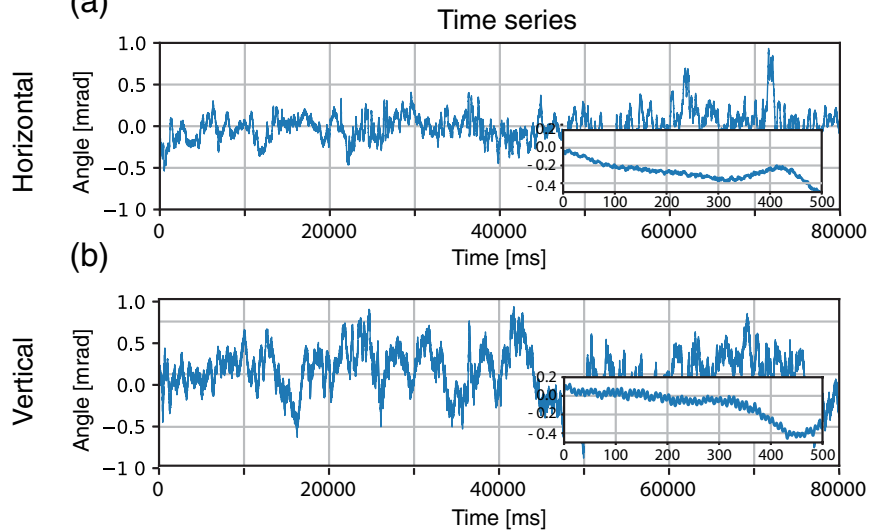

(c)

(d)
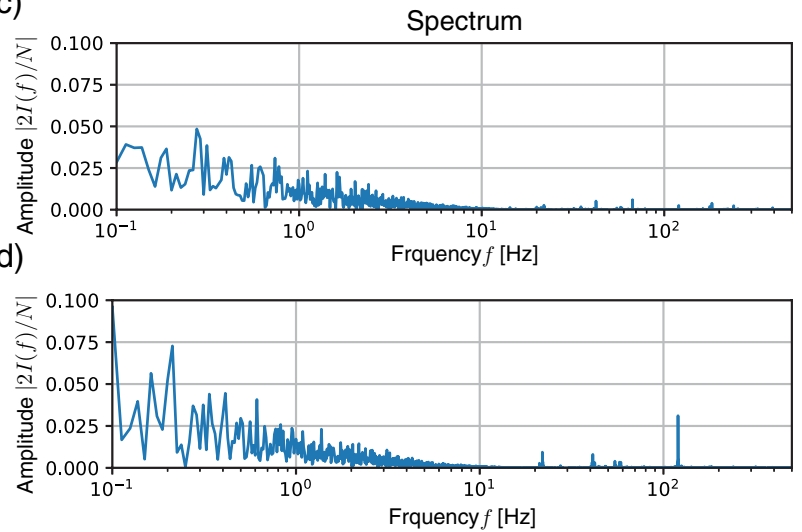

Figure 4. (a), (b) Time series of $1 \mathrm{kHz}$ non-amplified beam pointing angle at focus in horizontal and vertical directions, derived from the far-field mode captured by camera 2 and the near-field mode captured by camera 3. (c), (d) The amplitude $|2 I(f) / N|$ of the discrete Fourier transform $I(f)$ of the time series (a) and (b), respectively, highlighting the dominant components below $10 \mathrm{~Hz}$.

axis angle of the witness beam. The standard deviation was $\sigma_{\theta}=0.17 \mathrm{mrad}$ in horizontal direction and $0.22 \mathrm{mrad}$ in vertical direction. Note that these values are significantly higher than the angle-of-incidence fluctuations of the beams onto the OAP (position fluctuations on target translated into angle fluctuations assuming position on the OAP is fixed), and this difference is a direct result of the fact that optical axis of the focused laser beam is mainly dictated by position offset on the OAP surface (for example, a $0.3 \mathrm{~mm}$ position offset with no angle offset on the surface of the OAP with $3 \mathrm{~m}$ focal length results in a $0.1 \mathrm{mrad}$ delivered pointing angle). It turns out there is a high degree of correlation between the beam centroid on the OAP surface (camera 4) and pointing angle determined from the witness beam diagnostic, which is discussed in Section 6. Interestingly, compared with the time series of the beam centroids in Figure 3, the pointing angle fluctuations in Figure 4 are dominated by much lower frequencies, as is apparent in the zoomed-in insets of Figures 4(a) and 4(b). The discrete Fourier transform of the time series of the pointing angle fluctuations, calculated by replacing $c_{n}$ with $\theta_{n}$ in Equation (2), is shown in Figures 4(c) and 4(d). One can observe that the frequencies below $10 \mathrm{~Hz}$ are dominating the fluctuations of the pointing angles.

Another method of interpreting the Fourier spectra of the centroid and pointing angle fluctuations is by computing the cutoff-integrated power spectral density ${ }^{[27]}$. The relation between the time-series standard deviation $\sigma$ and the power spectral density $S(f)$ is

$$
\sigma=\sqrt{\int_{0}^{\infty} 2 S(f) \mathrm{d} f} .
$$

Thus, the standard deviation of the fluctuations $\sigma^{*}$ for $f$ less than $f_{\text {cutoff }}$ (for example, because the lower frequencies were eliminated with an active feedback system), can be calculated as

$$
\sigma^{*}\left(f_{\text {cutoff }}\right)=\sqrt{\int_{f_{\text {cutoff }}}^{\infty} 2 S(f) \mathrm{d} f .}
$$

Note that for $f_{\text {cutoff }}=0$, we retrieve $\sigma^{*}(0)=\sigma$. Using Equation (4), we calculated the cutoff-corrected standard deviation $\sigma^{*}$ for the centroid and angular time-series of Figures 3(a), 3(b), 4(a), and 4(b). Figure 5(a) shows the centroid standard deviation $\sigma^{*}$ as a function of cutoff frequency $f_{\text {cutoff }}$. The unfiltered standard deviation $\sigma^{*}(0)$ in horizontal and vertical directions was 5.0 and $6.5 \mu \mathrm{m}$, respectively, but drops as $f_{\text {cutoff }}$ is increased. As is evident, the standard deviation has strong contributions at less than $1 \mathrm{~Hz}$, around 20,40 , and $50-70 \mathrm{~Hz}$. If frequency components below $70 \mathrm{~Hz}$ were filtered out $\left(f_{\text {cutoff }}=70 \mathrm{~Hz}\right)$, the remaining standard deviation of the beam centroids would reduce to 1.0 and $0.81 \mu \mathrm{m}$, respectively, i.e., approximately a factor of five. In Figure 5(b), addressing the focal axis pointing angle, the unfiltered standard deviation $\sigma^{*}(0)$ in the horizontal and vertical directions was 0.17 and $0.22 \mathrm{mrad}$, respectively. One can observe the dominant contributions to be below $10 \mathrm{~Hz}$. Choosing $f_{\text {cutoff }}=10 \mathrm{~Hz}$ would reduce the standard deviation to 0.010 and $0.026 \mathrm{mrad}$, respectively. Note that these are the hypothetical examples and in reality the measured fluctuations cannot become zero when all the oscillations are eliminated by the active feedback system. To measure the limit of the position and angle fluctuations of the witness beam, a rigidly mounted single-mode fiber tip was placed at the witness beam focus location. Its position and angle fluctuations, which are the fluctuations of the diagnostics, were found to be $0.56 \mu \mathrm{m}$ in horizontal and $0.67 \mu \mathrm{m}$ in vertical direction, and $0.02 \mathrm{mrad}$ in horizontal and $0.03 \mathrm{mrad}$ in vertical direction, respectively. 

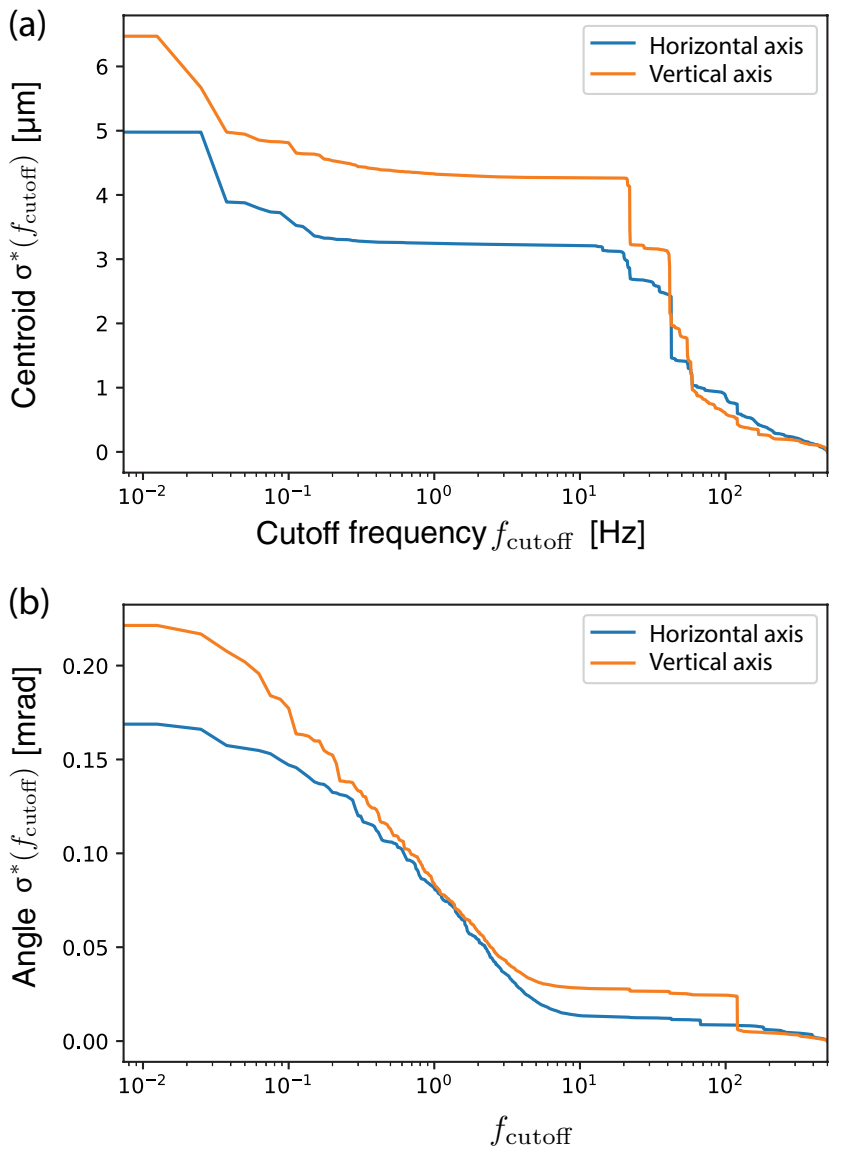

Figure 5. The standard deviation $\sigma^{*}$ of (a) the centroid fluctuations and (b) the angle fluctuations, with only frequencies above $f_{\text {cutoff }}$ included in the standard deviation calculation. This representation of the spectral content is a complimentary way to interpret the Fourier transforms of the time series in Figures 3 and 4.

\section{Main beam and witness beam centroid correlations}

If the $1 \mathrm{~Hz}$ amplified beam were to receive a transient position and angle perturbation, for example during the submillisecond pumping process in the Ti:sapphire crystals, it would simply not be appropriate to use the non-amplified pulse train as an active feedback driver. In the previous sections we characterized the correlation of the amplified $1 \mathrm{~Hz}$ high-power pulse on the mode imager (the main beam) and the witness beam setup (witness beam), and we used the nonamplified $1 \mathrm{kHz}$ pulse train to study the dominant spectral terms in the fluctuating position and angle at focus. Section 3 confirmed the witness beam as a correlated measurement of the main beam and Section 4 highlighted that most fluctuations are well below $70 \mathrm{~Hz}$. This opens a promising path to using the kilohertz pulse train for fast active feedback laser delivery stabilization. A further requirement is the correlation between the $1 \mathrm{~Hz}$ amplified pulse properties to the kilohertz pulse train.

To measure the correlation of the amplified beam to the non-amplified beam, we took advantage of the fact that the trigger timing for the witness beam cameras can be selected.
For example, for a given acquisition of the main amplified beam on camera 1, we triggered cameras 2 and 3 to record the non-amplified pulse $1 \mathrm{~ms}$ earlier. Note that in order not to damage the witness beam cameras while recording the non-amplified pulse, all attenuators were removed and a mechanical shutter with sub-millisecond close time was used to block the $1 \mathrm{~Hz}$ amplified beam. A schematic of the camera capture timings is shown in Figure 6(a). The Pearson's correlation coefficients $\rho$ for $N=100$ consecutive shots are shown in Figure 6(b), both in horizontal (left bars) and vertical directions (right bars). Two datasets are compared. The blue bars show $\rho$ when both camera 1 (mode imager) and camera 2 (witness beam) are timed to record the main (high-power) pulse (there was no delay on the camera 2 trigger, such that $\Delta t_{\text {cam }}=0 \mathrm{~ms}$ ). The orange bars show the case where $\Delta t_{\mathrm{cam}}=1 \mathrm{~ms}$, referring to the situation where camera 1 (mode imager) is recording the main (highpower) pulse, but camera 2 is recording the non-amplified pulse $1 \mathrm{~ms}$ earlier. The error bars show the standard error of the Pearson's correlation coefficient, $\sqrt{\left(1-\rho^{2}\right) /(N-2)^{[28]}}$. Note that for this dataset, the laser mode was not optimized as for the centroid data in Figure 2, which appears to manifest itself as a slightly reduced Pearson coefficient $\rho$ (small fluctuations in the wings of the laser mode will affect the centroid calculation).

Noteworthy from Figure 6(b) is the observation that there is no significant difference in the correlation coefficient $\rho$ for $\Delta t_{\mathrm{cam}}=0 \mathrm{~ms}$ and $\Delta t_{\mathrm{cam}}=1 \mathrm{~ms}$. This indicates that there is no significant random term in the transverse centroids as introduced by the amplification progress. This is a promising result when considering active feedback systems driven by the kilohertz non-amplified pulse train.

We also simulated how the Pearson coefficient $\rho$ would drop if we added to the data a deliberate centroid fluctuation only on the main (high-power) pulse. If a fluctuation following a Gaussian statistical distribution with $0.5 \mu \mathrm{m}$ standard deviation was added to mimic added centroid fluctuations in the amplified beam, the correlation $\rho$ at $\Delta t_{\text {cam }}=1 \mathrm{~ms}$ was found to drop by 0.1 (from 0.9 to 0.8 ) in both horizontal and vertical directions. The value further drops to 0.6-0.7 when the standard deviation is increased to $1.0 \mu \mathrm{m}$. As our experimental results in Figure 6(b) did not record a drop in $\rho$ exceeding the $\sim 0.05$ error bar, we can conclude that the additional focus position fluctuation during the amplification process is well below the error-bar-related upper bound of $<0.5 \mu \mathrm{m}$, which in turn is approximately $10 \%$ of the fluctuation of the main beam.

\section{Main beam and witness beam pointing angle correla- tions}

When addressing pointing angle, unfortunately, unlike the cameras 2 and 3 witness diagnostic, the mode imager setup 
(a)

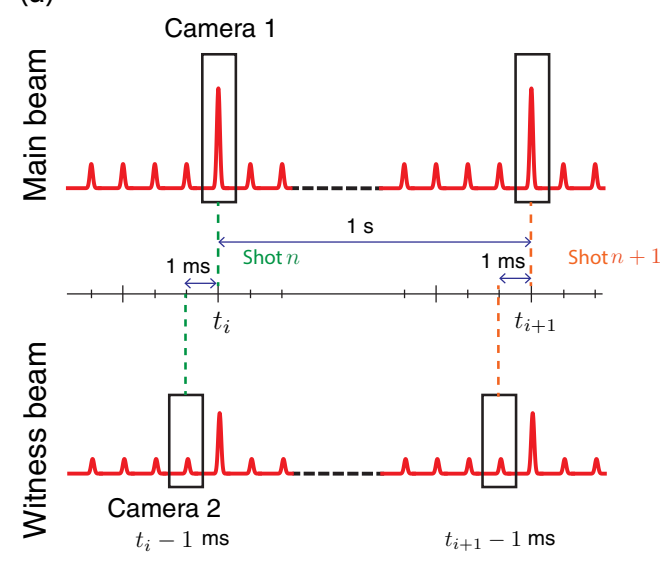

(b)

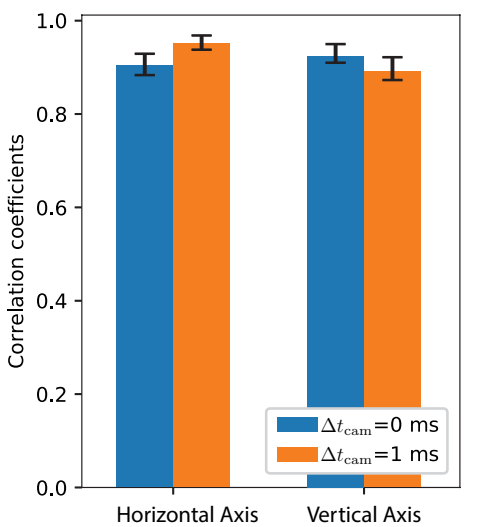

Figure 6. (a) Illustration of the main beam and the witness beam timing with respect to the camera capture exposure. The example shows the $\Delta t_{\text {cam }}=1$ ms case when camera 1 (mode imager) captures the $1 \mathrm{~Hz}$ amplified main beam, whereas camera 2 captures the non-amplified pulse $1 \mathrm{~ms}$ earlier. (b) Correlation coefficients $\rho$ derived from the beam centroids measured with cameras 1 and 2 , both for $\Delta t_{\text {cam }}=0 \mathrm{~ms}$ (blue bars) and $\Delta t_{\text {cam }}=1$ ms (orange bars). We observe no significant degradation in $\rho$ at $\Delta t_{\text {cam }}=1 \mathrm{~ms}$, indicating that the main pulse has not picked up a random centroid fluctuation during the amplification process.

(a)
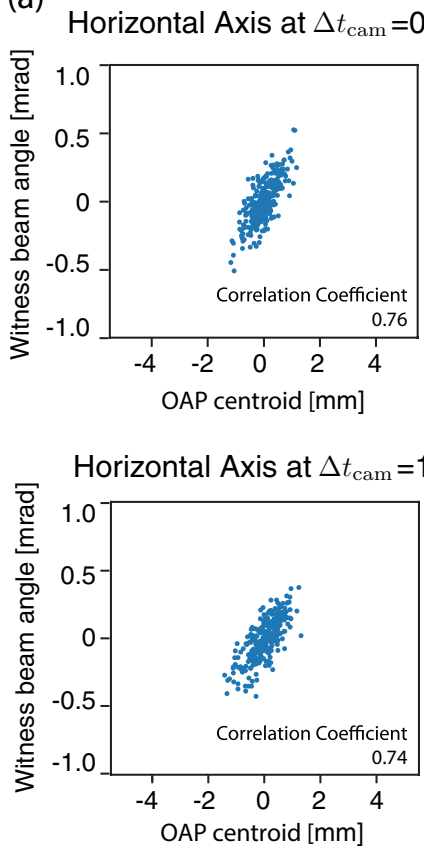
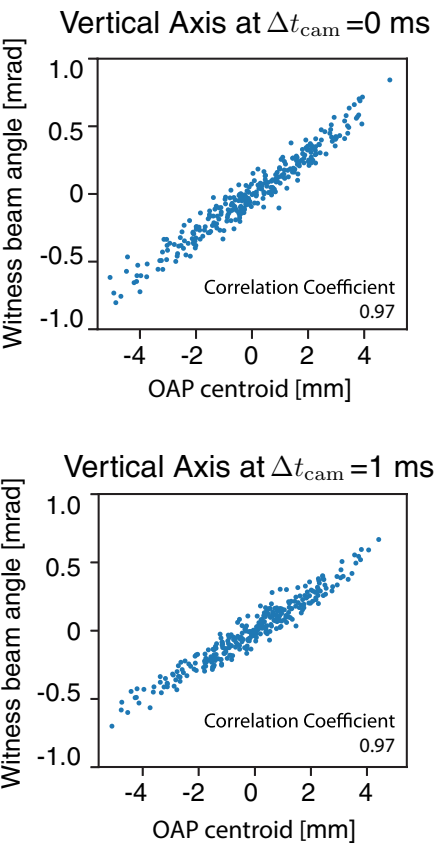

(b)

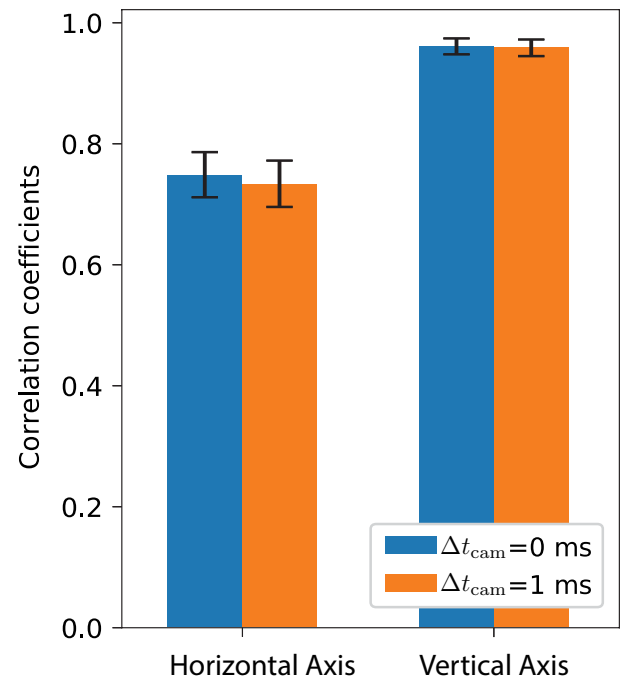

Figure 7. (a) Correlations of the main beam centroid at the OAP surface as captured with camera 4, and the pointing angle at focus captured with cameras 2 and 3 (witness beam diagnostic). Camera 4 always captured the main (high-power) $1 \mathrm{~Hz}$ pulses, whereas cameras 2 and 3 captured either the same pulse at $\Delta t_{\text {cam }}=0 \mathrm{~ms}$ or the non-amplified pulse $1 \mathrm{~ms}$ earlier at $\Delta t_{\text {cam }}=1 \mathrm{~ms}$. (b) Correlation coefficients $\rho$ for the plots in (a), showing that there are no significant differences in $\rho$ between $\Delta t_{\text {cam }}=0 \mathrm{~ms}$ and $\Delta t_{\text {cam }}=1 \mathrm{~ms}$, supporting the claim that the amplification process does not impart significant random fluctuations in the target-chamber laser axis angle.

did not have the ability to measure the pointing angle of the main beam at focus. However, we can still use the beam centroid location at the OAP surface, as measured by CCD camera 4 (Basler acA1300-22gm) in Figure 1, to estimate the amplified laser pointing angle near focus. Indeed, as the top plots in Figure 7(a) show, there is high degree of correlation between the beam centroid on the OAP surface (camera 4) and pointing angle determined from the witness beam diagnostic. The Pearson correlation coefficient $\rho$ is plotted as blue bars in Figure 7(b), for the case of $\Delta t_{\mathrm{cam}}=0 \mathrm{~ms}$ (both diagnostics recording the high-power main pulse). The standard deviation of the witness beam angle at $\Delta t_{\mathrm{cam}}=0$ was $0.16 \mathrm{mrad}$ in horizontal direction and $0.30 \mathrm{mrad}$ in transverse direction. The standard deviation of the centroid of the main beam at the OAP surface at $\Delta t_{\text {cam }}=0$ was $0.43 \mathrm{~mm}$ in horizontal direction and $2.0 \mathrm{~mm}$ in vertical 
direction. Offset of $0.43 \mathrm{~mm}$ in the OAP surface is equivalent to the angle offset of $0.14 \mathrm{mrad}$ at focus assuming no angle offset on the surface of the OAP with $3 \mathrm{~m}$ focal length. Note that the difference of the slope of the correlation between the horizontal axis and the vertical axis in Figure 7 is probably due to the existence of the non-symmetric high-order nearfield modes, and the investigation of this high-order content is beyond the scope of this paper.

To study the potential impact of the transient amplification process, we also executed a scan where the witness beam angle diagnostic recorded the pointing angle of the nonamplified pulse $1 \mathrm{~ms}$ earlier (at $\Delta t_{\text {cam }}=1 \mathrm{~ms}$ ), with the scatter plots shown at the bottom in Figure 7(a) and the correlation coefficient shown as orange bars in Figure 7(b). Just like with the centroid scans in Figure 6, there was no significant difference in Figure 7 between the correlation coefficients at $\Delta t_{\mathrm{cam}}=0 \mathrm{~ms}$ and $\Delta t_{\mathrm{cam}}=1 \mathrm{~ms}$. If a fluctuation following a Gaussian statistical distribution with $0.01 \mathrm{mrad}$ standard deviation was added to mimic the angle fluctuations in the amplified beam, the correlation coefficient $\rho$ drops below the standard error of the measured value. This suggests that pointing angle at focus is degraded by no more than $0.01 \mathrm{mrad}$ of additional pointing angle fluctuation from the amplification process, i.e., approximately $5 \%$ of the main beam pointing angle fluctuation.

\section{Conclusion}

An online non-perturbative monitor of the high-power laser delivery was installed on a $1-\mathrm{Hz} 100-\mathrm{TW}$-class laser system, measuring both the transverse focus position and the pointing angle at focus. The diagnostic was based on a unique double-surface-coated wedged-mirror used as a final steering mirror, generating a highly correlated witness beam. Correlation at high-power operation was verified with a mode imager far-field camera.

In addition to $1 \mathrm{~Hz}$ high-power pulses, the $1 \mathrm{kHz}$ nonamplified pulse train was also sent to the witness beam cameras. The $1 \mathrm{kHz}$ non-amplified witness beam is well suited as an alignment laser, because it propagates with the amplified beam from the oscillator all the way up to the last reflection at the final steering mirror, with its beam size and the divergence matched with the amplified beam. By performing a Fourier analysis on the centroid and pointing angle time series, the spectra of the position and the angle fluctuations were characterized. Although the pointing angle fluctuations were dominated by environmental drivers below $10 \mathrm{~Hz}$, the centroid fluctuations consisted both of lowfrequency $(<1 \mathrm{~Hz})$ variations and various discrete contributions around 20,40 , and $50-70 \mathrm{~Hz}$. By varying the camera trigger timing, it was verified that the amplified $1 \mathrm{~Hz}$ laser pulses did not accumulate random position and angle fluctuations during the amplification process by more than $0.5 \mu \mathrm{m}$ and $0.01 \mathrm{mrad}$, respectively.
The various observations presented in this paper support the future concept that a less than $70 \mathrm{~Hz}$ bandwidth active feedback system, using the kilohertz non-amplified pulse train as sampling source, is sufficient to non-perturbatively stabilize the position and pointing angle of the 1-Hz-class high-power laser pulses by approximately a factor of five. This would present a significant advance in the stability and tunability of high-power laser applications such as laserplasma accelerators, among others.

\section{Acknowledgments}

This work was supported by the U.S. Department of Energy (DOE), Office of Science, the Office of Basic Energy Sciences, and the Office of High Energy Physics, under Contract No. DE-AC02-05CH11231, and the Gordon and Betty Moore Foundation under Grant No. GBMF4898. The authors would like to thank Zac Eisentraut, Mark Kirkpatrick, Arturo Magana, Greg Mannino, Joe Riley, Tyler Sipla, and Nathan Ybarrolaza for technical support.

\section{References}

1. M. Pittman, S. Ferré, J.-P. Rousseau, L. Notebaert, J.-P. Chambaret, and G. Chériaux, Appl. Phys. B 74, 529 (2002).

2. K. Nakamura, H.-S. Mao, A. J. Gonsalves, H. Vincenti, D. E. Mittelberger, J. Daniels, A. Magana, C. Toth, and W. P. Leemans, IEEE J. Quantum Electron. 53, 1200121 (2017).

3. C. N. Danson, C. Haefner, J. Bromage, T. Butcher, J.-C. F. Chanteloup, E. A. Chowdhury, A. Galvanauskas, L. A. Gizzi, J. Hein, D. I. Hillier, N. W. Hopps, Y. Kato, E. A. Khazanov, R. Kodama, G. Korn, R. Li, Y. Li, J. Limpert, J. Ma, C. H. Nam, D. Neely, D. Papadopoulos, R. R. Penman, L. Qian, J. J. Rocca, A. A. Shaykin, C. W. Siders, C. Spindloe, S. Szatmári, R. M. G. M. Trines, J. Zhu, P. Zhu, and J. D. Zuegel, High Power Laser Sci. Eng. 7, e54 (2019).

4. Z. Zhang, F. Wu, J. Hu, X. Yang, J. Gui, P. Ji, X. Liu, C. Wang, Y. Liu, X. Lu, Y. Xu, Y. Leng, R. Li, and Z. Xu, High Power Laser Sci. Eng. 8, e4 (2020).

5. D. Strickland and G. Mourou, Opt. Commun. 56, 219 (1985).

6. A. J. Gonsalves, K. Nakamura, J. Daniels, C. Benedetti, C. Pieronek, T. C. H. de Raadt, S. Steinke, J. H. Bin, S. S. Bulanov, J. van Tilborg, C. G. R. Geddes, C. B. Schroeder, C. Toth, E. Esarey, K. Swanson, L. Fan-Chiang, G. Bagdasarov, N. Bobrova, V. Gasilov, G. Korn, P. Sasorov, and W. P. Leemans, Phys. Rev. Lett. 122, 084801 (2019).

7. K. T. Phuoc, S. Corde, C. Thaury, V. Malka, A. Tafzi, J.-P. Goddet, R. Shah, S. Sebban, and A. Rousse, Nat. Photonics 6, 308 (2012).

8. A. R. Maier, N. Kajumba, A. Guggenmos, C. Werle, J. Wenz, N. Delbos, B. Zeitler, I. Dornmair, J. Schmidt, E. M. Gullikson, F. Krausz, U. Schramm, U. Kleineberg, S. Karsch, and F. Gruner, Sci. Rep. 10, 5634 (2020).

9. M. Schnell, A. Sävert, B. Landgraf, M. Reuter, M. Nicolai, O. Jäckel, C. Peth, T. Thiele, O. Jansen, A. Pukhov, O. Willi, M. C. Kaluza, and C. Spielmann, Phys. Rev. Lett. 108, 075001 (2012).

10. F. Isono, J. van Tilborg, S. Barber, C. Geddes, H.-E. Tsai, C. Schroeder, and W. P. Leemans, in CLEO: Science and Innovations (2019), paper SF3I.1. 
11. P. Zeitoun, G. Faivre, S. Sebban, T. Mocek, A. Hallou, M. Fajardo, D. Aubert, P. Balcou, F. Burgy, D. Douillet, S. Kazamias, G. de Lachèze-Murel, T. Lefrou, S. le Pape, P. Mercère, H. Merdji, A. S. Morlens, J. P. Rousseau, and C. Valentin, Nature 431, 426 (2004).

12. S. Steinke, J. H. Bin, J. Park, Q. Ji, K. Nakamura, A. J. Gonsalves, S. S. Bulanov, M. Thèvenet, C. Toth, J.-L. Vay, C. B. Schroeder, C. G. R. Geddes, E. Esarey, T. Schenkel, and W. P. Leemans, Phys. Rev. Accel. Beams 23, 021302 (2020).

13. C. Geddes, C. Toth, J. Van Tilborg, E. Esarey, C. Schroeder, J. Cary, and W. Leemans, Phys. Rev. Lett. 95, 145002 (2005).

14. C. Rechatin, J. Faure, A. Ben-Ismaïl, J. Lim, R. Fitour, A. Specka, H. Videau, A. Tafzi, F. Burgy, and V. Malka, Phys. Rev. Lett. 102, 164801 (2009).

15. L.-L. Yu, E. Esarey, C. Schroeder, J.-L. Vay, C. Benedetti, C. Geddes, M. Chen, and W. Leemans, Phys. Rev. Lett. 112, 125001 (2014).

16. B. Hidding, G. Manahan, T. Heinemann, P. Scherkl, F. Habib, D. Ullmann, A. Beaton, A. Sutherland, A. Knetsch, O. Karger, G. Wittig, B. O'Shea, V. Yakimenko, M. Hogan, S. Green, C. Clarke, S. Gessner, J. Rosenzweig, A. Deng, M. Litos, D. Bruhwiler, J. Smith, J. Cary, R. Zgadzaj, M. C. Downer, C. Lindstrøm, E. Adli, and G. Andonian, in 8th International Particle Accelerator Conference (2017), p. 1252.

17. A. Gonsalves, K. Nakamura, C. Lin, J. Osterhoff, S. Shiraishi, C. Schroeder, C. Geddes, C. Tóth, E. Esarey, and W. Leemans, Phys. Plasmas 17, 056706 (2010).

18. B. Miao, L. Feder, J. Shrock, A. Goffin, and H. Milchberg, Phys. Rev. Lett. 125, 074801 (2020).

19. G. Genoud, F. Wojda, M. Burza, A. Persson, and C.-G. Wahlstrom, Rev. Sci. Instrum. 82, 033102 (2011).

20. S. J. D. Dann, C. D. Baird, N. Bourgeois, O. Chekhlov, S. Eardley, C. D. Gregory, J.-N. Gruse, J. Hah, D. Hazra, S. J. Hawkes, C. J. Hooker, K. Krushelnick, S. P. D. Mangles, V. A. Marshall, C. D. Murphy, Z. Najmudin, J. A. Nees, J. Osterhoff, B. Parry, P. Pourmoussavi, S. V. Rahul, P. P. Rajeev, S. Rozario, J. D. E. Scott, R. A. Smith, E. Springate, Y. Tang, S. Tata, A. G. R. Thomas, C. Thornton, D. R. Symes, and M. J. V. Streeter, Phys. Rev. Accel. Beams 22, 041303 (2019).

21. A. R. Maier, N. M. Delbos, T. Eichner, L. Hübner, S. Jalas, L. Jeppe, S. W. Jolly, M. Kirchen, V. Leroux, P. Messner, M. Schnepp, M. Trunk, P. A. Walker, C. Werle, and P. Winkler, Phys. Rev. X 10, 031039 (2020).

22. W. Leemans, "Laser technology for k-BELLA and beyond," Technical Report (Lawrence Berkeley National Laboratory, 2017).
23. M. K. Weikum, T. Akhter, D. Alesini, A. S. Alexandrova, M. P. Anania, N. E. Andreev, I. A. Andriyash, A. Aschikhin, R. W. Assmann, T. Audet, A. Bacci, I. F. Barna, A. Beaton, A. Beck, A. Beluze, A. Bernhard, S. Bielawski, F. G. Bisesto, F. Brandi, R. Brinkmann, E. Bruendermann, M. Büscher, M. H. Bussmann, G. Bussolino, A. Chance, M. Chen, E. Chiadroni, A. Cianchi, J. A. Clarke, J. Cole, M. E. Couprie, M. Croia, B. Cros, P. A. Crump, G. Dattoli, A. D. Dotto, N. Delerue, S. D. Nicola, J. M. Dias, U. Dorda, R. Fedele, A. F. Pousa, M. Ferrario, F. Filippi, G. Fiore, R. A. Fonseca, M. Galimberti, A. Gallo, A. Ghaith, D. Giove, A. Giribono, L. A. Gizzi, F. J. Grüner, A. F. Habib, C. Haefner, T. Heinemann, B. Hidding, B. J. Holzer, S. M. Hooker, T. Hosokai, M. Huebner, A. Irman, F. J. Jafarinia, D. A. Jaroszynski, C. Joshi, M. Kaluza, M. Kando, O. S. Karger, S. Karsch, E. Khazanov, D. Khikhlukha, A. Knetsch, D. Kocon, P. Koester, O. S. Kononenko, G. Korn, I. Kostyukov, K. O. Kruchinin, L. Labate, C. L. Blanc, C. Lechner, W. Leemans, A. Lehrach, X. Li, V. Libov, A. Lifschitz, V. Litvinenko, W. Lu, O. Lundh, A. R. Maier, V. Malka, G. G. Manahan, S. P. D. Mangles, B. Marchetti, A. M. de la Ossa, J. L. Martins, P. D. Mason, F. Massimo, F. Mathieu, G. Maynard, Z. Mazzotta, A. Y. Molodozhentsev, A. Mostacci, A. S. Mueller, C. D. Murphy, Z. Najmudin, P. A. P. Nghiem, F. Nguyen, P. Niknejadi, J. Osterhoff, D. O. Espinos, D. N. Papadopoulos, B. Patrizi, V. Petrillo, M. A. Pocsai, K. Poder, R. Pompili, L. Pribyl, D. Pugacheva, P. P. Rajeev, S. Romeo, M. R. Conti, A. R. Rossi, R. Rossmanith, E. Roussel, A. A. Sahai, G. Sarri, L. Schaper, P. Scherkl, U. Schramm, C. B. Schroeder, J. Scifo, L. Serafini, Z. M. Sheng, C. Siders, L. O. Silva, T. Silva, C. Simon, U. Sinha, A. Specka, M. J. V. Streeter, E. N. Svystun, D. Symes, C. Szwaj, G. E. Tauscher, D. Terzani, N. Thompson, G. Toci, P. Tomassini, R. Torres, D. Ullmann, C. Vaccarezza, M. Vannini, J. M. Vieira, F. Villa, C. G. Wahlstrom, R. Walczak, P. A. Walker, K. Wang, C. P. Welsch, S. M. Wiggins, J. Wolfenden, G. Xia, M. Yabashi, J. Zhu, and A. Zigler, J. Phys. Conf. Ser. 1350, 012059 (2019).

24. J. van Tilborg, S. K. Barber, F. Isono, C. B. Schroeder, E. Esarey, and W. P. Leemans, AIP Conf. Proc. 1812, 020002 (2017).

25. https://www.coherent.com/.

26. https://www.thalesgroup.com/en.

27. J. L. Stanford and S. B. Vardeman, Statistical Methods for Physical Science (Academic Press, 1994).

28. J. Cohen, P. Cohen, S. G. West, and L. S. Aiken, Applied Multiple Regression/Correlation Analysis for the Behavioral Sciences (Lawrence Erlbaum Associates, 2003). 